\title{
La pauvreté en Allemagne : évolution, structure et disparités régionales
}

Poverty in Germany: Development, structure and regional disparities

Armut in Deutschland : Entwicklung, Struktur und regionale Disparitäten

\section{Britta Klagge}

Traducteur : Michel Deshaies

\section{(2) OpenEdition \\ Journals}

Édition électronique

URL : http://journals.openedition.org/rge/3986

DOI : $10.4000 /$ rge.3986

ISSN : 2108-6478

Éditeur

Association des géographes de l'Est

Édition imprimée

Date de publication : 1 janvier 2001

ISSN : 0035-3213

Référence électronique

Britta Klagge, "La pauvreté en Allemagne : évolution, structure et disparités régionales », Revue Géographique de l'Est [En ligne], vol. 41 / 1-2 | 2001, mis en ligne le 25 juillet 2013, consulté le 08 septembre 2020. URL : http://journals.openedition.org/rge/3986 ; DOI : https://doi.org/10.4000/rge 3986

Ce document a été généré automatiquement le 8 septembre 2020

Tous droits réservés 


\title{
La pauvreté en Allemagne : évolution, structure et disparités régionales
}

\author{
Poverty in Germany : Development, structure and regional disparities \\ Armut in Deutschland : Entwicklung, Struktur und regionale Disparitäten
}

\section{Britta Klagge}

Traduction : Michel Deshaies

\section{Introduction}

1 Dans les états industrialisés, la pauvreté absolue qui menace l'existence physique ne joue pratiquement plus de rôle. Aussi, les recherches sur les situations de pauvreté s'intéressent-elles en général à la pauvreté «relative». Le Conseil de l'Union européenne définit comme étant en situation de pauvreté relative les personnes et les ménages qui « disposent de moyens (matériels, culturels et sociaux) si faibles qu'ils sont exclus du mode de vie considéré comme étant un niveau minimum dans l'état-membre où ils vivent " (d'après Geißler, 1996). Le concept de pauvreté relative est donc variable en fonction de la société et de l'époque.

En Allemagne et dans les autres pays industrialisés, l'évolution économique et sociale des 30 dernières années est marquée par de profondes mutations structurelles, et en particulier, depuis les années 80, le nombre de pauvres (relatifs) a fortement augmenté. Ce sont en premier lieu les changements structurels de l'économie et de l'emploi qui sous-tendent cette évolution. Dans ce contexte, la forte montée du chômage, la part croissante des situations d'emploi précaire par rapport aux actifs bénéficiant de contrats à durée indéterminée ont une importance toute particulière. La proportion croissante de personnes seules et de ménages monoparentaux est un facteur supplémentaire puisque, dans le cas d'une perte de revenus (par exemple dans une 
situation de chômage), les mécanismes compensatoires ne peuvent pas jouer beaucoup à l'intérieur du ménage.

3 On considère généralement que l'accentuation des différences sociales s'accompagne d'une plus forte différenciation spatiale de la population, notamment en ce qui concerne ses caractéristiques sociales; en d'autres termes que les disparités spatiales augmentent avec l'accroissement des inégalités sociales. Ce processus peut se produire au moins à trois échelles spatiales :

1. celle des inégalités entre les grands ensembles régionaux; en Allemagne en particulier, les différences entre l'ouest et l'est ainsi qu'entre le nord et le sud;

2. celle de l'accentuation des différences entre les régions urbaines et les régions rurales, et en particulier entre la ville et les espaces environnants (comme conséquence de la suburbanisation des classes à revenus moyens-supérieurs) ;

3. selon les disparités à l'intérieur des villes, entre les quartiers d'une même ville.

Dans cet article sont présentés les résultats d'analyses empiriques réalisées sur les deux premières échelles spatiales ${ }^{1}$. Les données de base sont constituées par l'importance des aides sociales sous la forme des densités d'aide sociale. La densité d'aide sociale correspond au nombre de personnes pour 1000 habitants bénéficiant d'aides destinées à assurer la vie quotidienne, à l'exclusion de celles se trouvant dans des structures d'accueil. Cette forme d'aide est versée lorsque les revenus du ménage sont inférieurs à un certain montant (correspondant à un niveau de vie spécifique) permettant de subvenir aux besoins ordinaires comme la nourriture, l'habillement et le logement, y compris le chauffage, les biens mobiliers et d'autres besoins de la vie quotidienne.

Cette définition de la pauvreté n'est pas sans poser des problèmes puisqu'elle ne mesure que la pauvreté financière et donc seulement la pauvreté soulagée par l'État. C'est pourquoi, dans la littérature récente, on prend en compte non seulement l'insuffisance des ressources financières, mais aussi d'autres situations d'insuffisance comme par exemple dans les domaines de l'habitat, de la santé, de la formation et des relations sociales (sur les concepts pluridimensionnels de la pauvreté, voir Hartmann 1981, Hanesch \& al. 1994, Leibfried \& al. 1995, Geißler 1996). Mais, par contraste avec le niveau d'aide sociale, les notions de pauvreté reposant sur le concept des multiples situations d'insuffisance sont difficiles à appréhender statistiquement. Celà est valable également pour certaines définitions de la pauvreté basées sur le niveau de ressource, pour lesquelles la pauvreté est définie à partir d'un niveau de ressource oscillant autour d'un revenu moyen des ménages ( $40 \%, 50 \%$ ou $60 \%$ des valeurs seuils). Dans celles-ci en effet, le problème des « chiffres noirs » de la pauvreté, c'est-à-dire de la "pauvreté cachée », n'est pas pris en compte. Malgré le problème que pose le fait d'assimiler la pauvreté soulagée par l'État à la pauvreté effective, les données sur l'aide sociale constituent néanmoins la meilleure source d'information, en particulier pour les comparaisons entre régions, car elles sont disponibles partout et permettent une différenciation spatiale.

En ce qui concerne l'interprétation des données sur l'aide sociale, on doit par ailleurs prendre en compte quelques points supplémentaires résultant, entre autres, du réajustement des statistiques sur l'aide sociale de l'année 1994 (Neuhauser 1996). Tandis que, jusqu'en 1993 les données concernant en particulier les classes démographiques n'étaient relevées que sous la forme de données annuelles cumulées, à partir de 1994 on réalise une comptabilité journalière. Les valeurs des deux types de relevés ne sont comparables que sous certaines conditions; les séries basées sur les 
données annuelles cumulées se terminent en $1993^{2}$. En outre, depuis la fin de l'année 1993 et l'entrée en vigueur de la nouvelle loi sur les prestations accordées aux demandeurs d'asile, ceux-ci obtiennent de plus faibles prestations et sont ainsi retranchés des statistiques sur l'aide sociale ${ }^{3}$. Enfin, dans l'analyse des données régionales à partir de 1990, il faut prendre en compte la position différente de Berlin suivant qu'on la considère comme faisant partie de l'Allemagne de l'Ouest (BerlinOuest) et/ou de par sa localisation, de l'Allemagne de l'Est.

\section{L'aide sociale en Allemagne}

7 Le nombre des bénéficiaires de l'aide sociale en Allemagne de l'Ouest (en incluant Berlin-ouest) a quadruplé de 1970 à 1998. En 1998, il y avait au total en Allemagne de l'Ouest et de l'Est 2,9 millions de bénéficiaires de l'aide sociale, parmi lesquels plus d'un tiers étaient des enfants (valeurs à chaque fois à la fin de l'année). Dans ce qui suit, on présentera l'évolution des densités de l'aide sociale ainsi que le changement du risque de paupérisation dans des classes démographiques sélectionnées et on discutera des facteurs importants influençant ces évolutions.

\section{A. Évolution depuis 1970}

8 Depuis 1970 la densité d'aide sociale a continuellement augmenté, à l'exception de quelques années (1977-1981, 1990-1991). Une comparaison des courbes d'évolution montre que l'une des principales causes de ce développement est l'accroissement du chômage (fig.1). Les phases de récession avec une forte croissance du chômage (1973-1975, 1980-1983) sont toujours suivies d'une phase où l'augmentation des bénéficiaires de l'aide sociale est supérieure à la moyenne (1973-1977, 1981-1989). Comme seul le chômage de longue durée conduit avec un temps de retard (après épuisement des premières prestations, comme l'allocation et l'aide au chômage) à la perception de l'aide sociale, la courbe de la pauvreté se tient à un niveau inférieur et présente de moins fortes variations que la courbe du chômage. 
Figure 1 : Évolution des taux de chômage (a) et des densités d'aide sociale (b) en Allemagne (c) à partir de 1970 et 1991

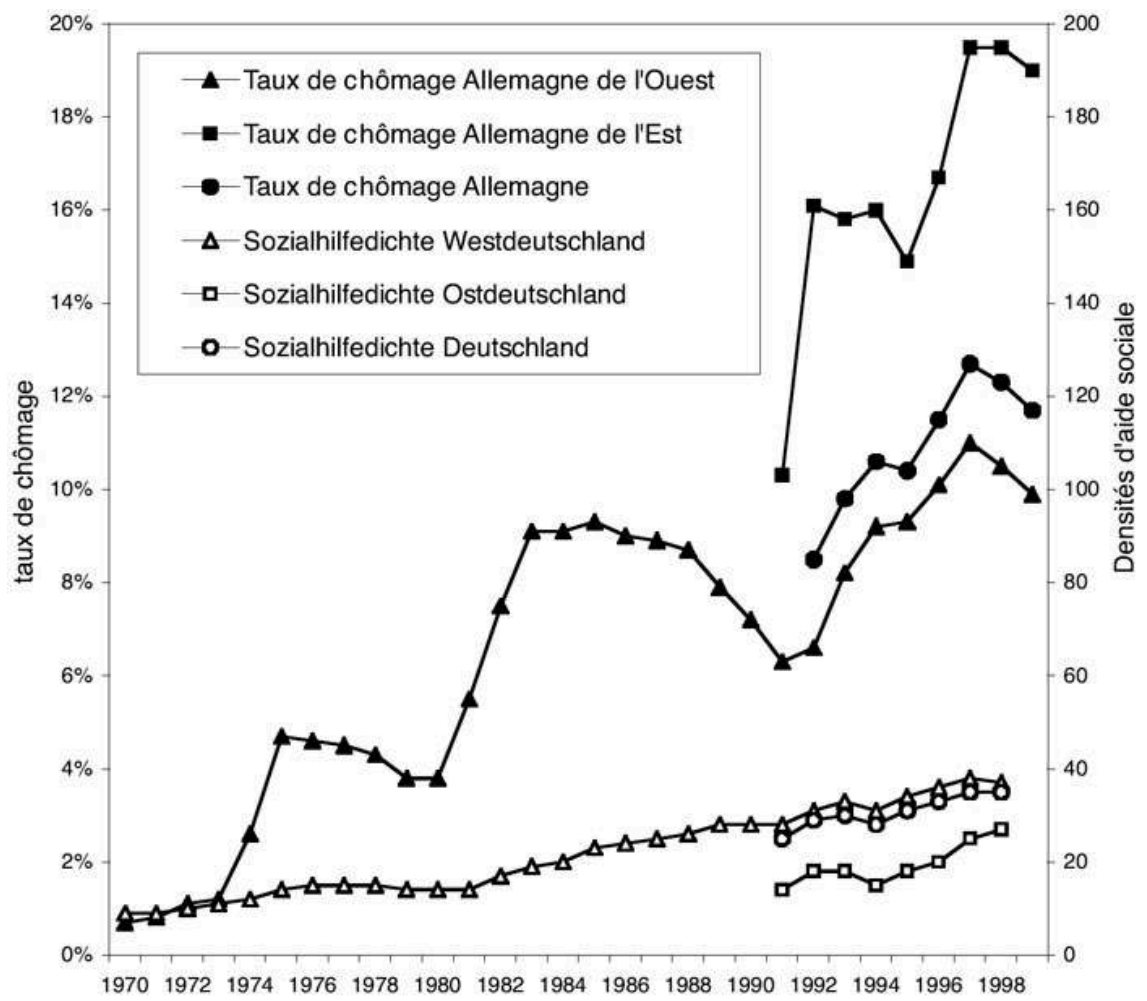

(a) Valeurs moyennes annuelles; (b) Valeurs à la fin de l'année (incluant les demandeurs d'asile jusqu'en 1993) pour $1000 \mathrm{~h}$; (c) Allemagne de l'Ouest incluant Berlin-ouest, Allemagne de l'Est sans Berlin-Ouest.

Sources : Bundesanstalt für Arbeit, Statistisches Bundesamt.

9 L'évolution à partir de 1989 est marquée par la réunification et les mutations économiques et démographiques qui lui sont liées. Entre 1991 et 1997, on peut observer une nouvelle montée de la densité d'aide sociale qui suit un cours largement parallèle à l'évolution du marché du travail. L'évolution actuelle à partir de 1997 montre, au moins pour l'Allemagne de l'Ouest, un trend positif qui, eu égard à la diminution du taux de chômage, pourrait se poursuivre. Avec une valeur de 35 pour 1000 habitants à la fin de l'année 1998, l'Allemagne de l'Ouest (avec Berlin-ouest) présente toutefois une densité d'aide sociale nettement plus élevée que l'Allemagne de l'Est (sans Berlin-ouest) où elle n'atteint que 27. Les différences entre l'Allemagne de l'Est et l'Allemagne de l'Ouest sont plus précisément expliquées dans le paragraphe III. A.

\section{B. Impact sur les différentes classes d'âge}

Entre 1970 et 1993 les relations entre les densités d'aide sociale des différentes classes d'âge en Allemagne de l'Ouest se sont notablement modifiées, bien que, dans toutes les classes d'âge (à l'exception des plus de 65 ans), la part des bénéficiaires de l'aide sociale ait augmenté (fig. 2, pour la situation actuelle voir fig. 4). Au début des années 70, c'étaient les personnes âgées, surtout les femmes n'ayant aucun droit à la retraite, qui constituaient la classe d'âge présentant le plus fort risque de paupérisation. Depuis lors, l'amélioration de la garantie vieillesse, en particulier la revalorisation des retraites, a 
permis de réduire (relativement) la paupérisation des personnes âgées ; aujourd'hui, les personnes âgées constituent la classe d'âge la moins atteinte par la pauvreté.

Figure. 2 : Évolution des densités d'aide sociale (a) des différentes classes d'âge en comparaison de la moyenne en Allemagne de l'Ouest (avec Berlin-ouest) de 1970 à 1993.

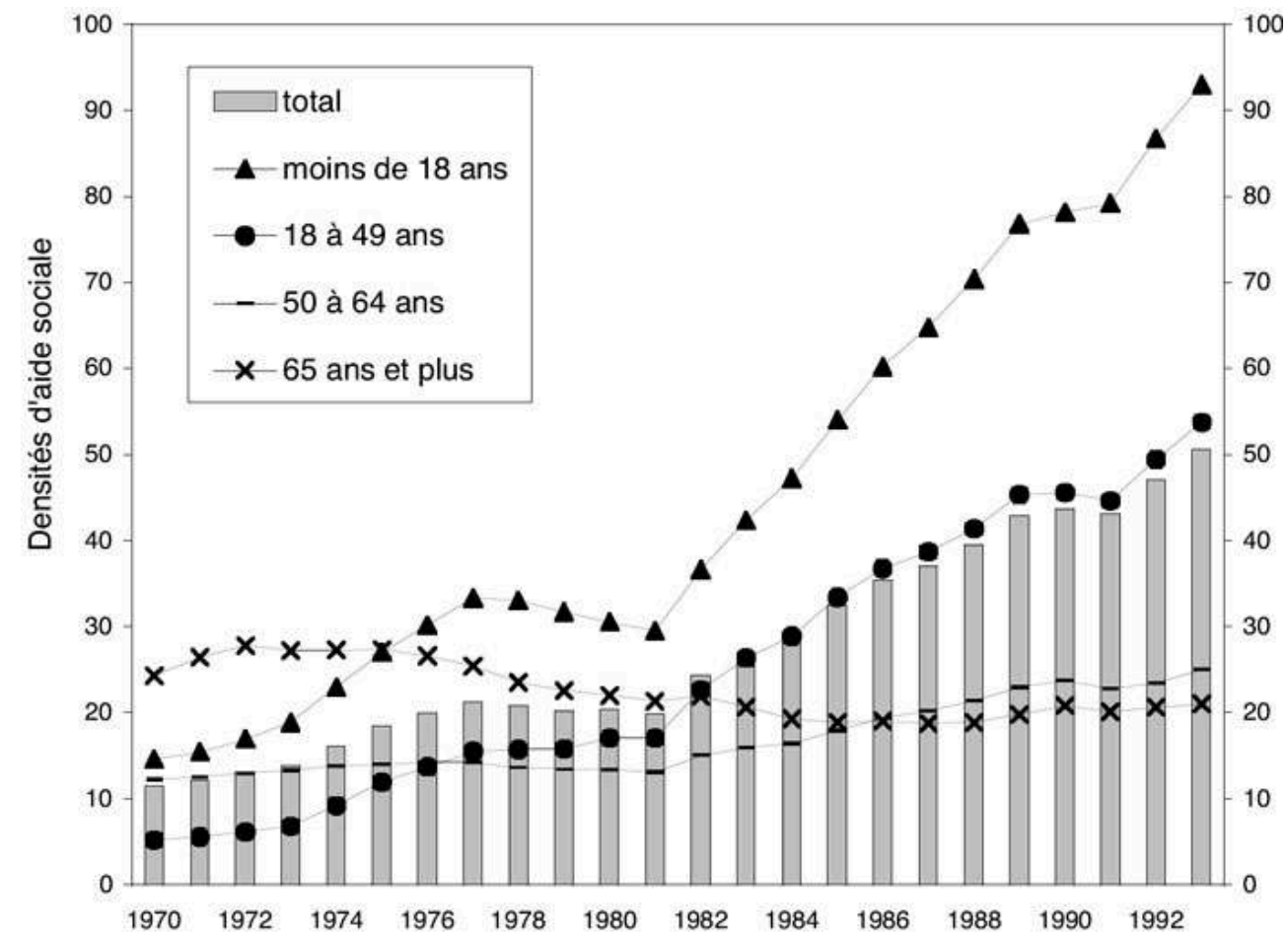

(a) Valeurs annuelles cumulées

Source : Statistisches Bundesamt

11 Par contre, on enregistre une forte augmentation des bénéficiaires de l'aide sociale chez les enfants (moins de 18 ans). En Allemagne de l'Ouest, il y a en moyenne, depuis le début des années 90 , plus de 80 enfants pour 1000 qui ont touché une aide sociale au moins une fois dans l'année. Les enfants sont ainsi aujourd'hui la classe d'âge la plus fortement atteinte par la pauvreté; celà concerne en particulier les enfants qui grandissent au sein d'un ménage monoparental, en général avec leur mère (Biebczack, Milz 1995). En-dehors des ménages avec beaucoup d'enfants (trois et plus), les ménages monoparentaux constituent le type de ménage présentant le plus grand risque de paupérisation. Ce type de ménage est particulièrement atteint par la pauvreté puisque le parent élevant seul ses enfants, en l'occurrence la mère, ne peut exercer qu'une activité limitée en raison même de la charge des enfants. De plus, dans beaucoup de cas, l'autre parent, en l'occurrence le père, ne parvient pas, ou seulement partiellement, à satisfaire l'obligation de verser une pension alimentaire.

\section{La paupérisation des étrangers}

Tandis que, dans les années 70, les étrangers présentaient une densité d'aide sociale plus faible que celle de la population allemande, depuis 1980 elle a complètement " décollé " par rapport à celle des Allemands (fig. 3). Un problème général est que, dans les statistiques de l'aide sociale, on ne distingue pas entre les différentes catégories 
d'étrangers. A la fin des années 80 et au début des années 90, les demandeurs d'asile représentaient une grande partie des étrangers bénéficiaires de l'aide sociale et constituaient ainsi un facteur important expliquant les forts taux d'augmentation de la densité d'aide sociale des étrangers (Wendt 1995, Klagge 1998).

Figure 3 : Comparaison de l'évolution des densités d'aide sociale (a) chez les étrangers, les Allemands et dans l'ensemble de la population - avec en arrière-plan le nombre d'étrangers (b) en Allemagne de l'Ouest (avec Berlin-ouest) pour la période 1970-1993

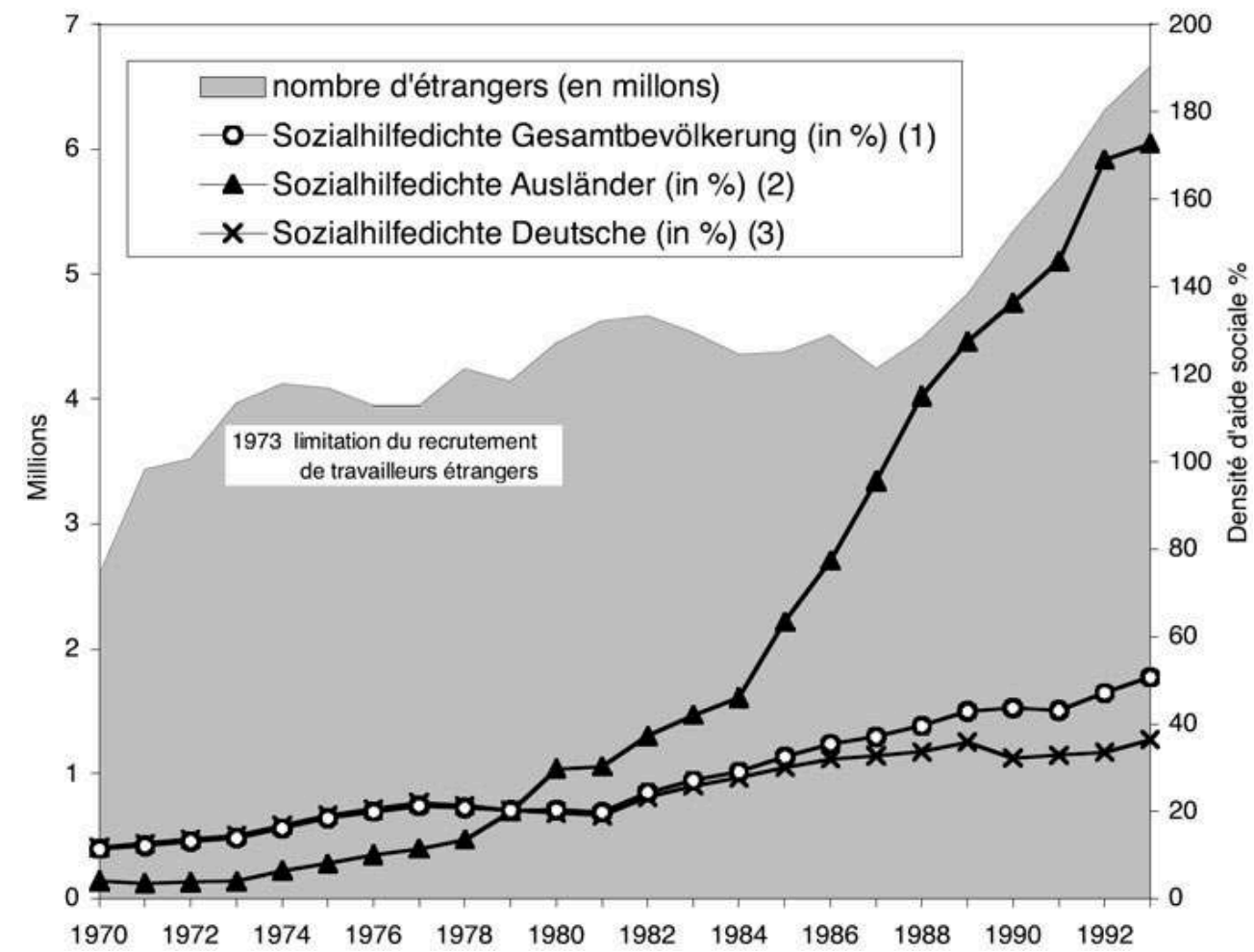

(a) Données annuelles cumulées; (b) A partir de 1991 le nombre d'étrangers pour l'Allemagne de l'Ouest inclut Berlin-est puisque les étrangers de la ville anciennement divisée sont recensés de façon globale. Comme cependant le nombre d'étrangers à Berlin-est est très faible en comparaison de Berlin-ouest, cela ne pose pas de problèmes; (1) Ensemble de la population; (2) Étrangers ; (3) Allemands.

Source : Bundesministerium des Inneren, Statistisches Bundesamt.

Les données actuelles, dans lesquelles les demandeurs d'asile ne sont plus pris en compte, montrent cependant que le risque de paupérisation des étrangers en Allemagne est toujours beaucoup plus élevé que dans la population allemande (densité d'aide sociale de 91 pour les étrangers contre 30 pour les Allemands, données de la fin de l'année 1998). Des recherches plus précises montrent toutefois qu'eu égard à la perception de l'aide sociale, les étrangers présentent les mêmes caractéristiques que la population allemande, c'est-à-dire qu'ils obtiennent l'aide sociale pour des raisons semblables. Les étrangers sont plus touchés que les Allemands par le chômage, ont en proportion beaucoup d'enfants et sont en conséquence fortement surreprésentés dans les groupes à fort risque de paupérisation (Buchel et al. 1997). 


\section{Les disparités régionales de perception de l'aide sociale}

En ce qui concerne la répartition régionale de la pauvreté et des versements de l'aide sociale, il existe des préjugés généraux sur le gradient nord-sud et sur la pauvreté en Allemagne de l'Est par rapport à l'Allemagne de l'Ouest. Afin de comprendre globalement le phénomène, il faut s'intéresser de plus près au contexte et à l'expression des contrastes à grande et à petite échelle. En dehors des disparités entre l'est et l'ouest et entre le nord et le sud, on considèrera dans ce qui va suivre le contraste ville-campagne.

\section{A. Comparaison entre l'Est et l'Ouest}

Depuis le 1er janvier 1991 les habitants de l'Allemagne de l'Est peuvent aussi recevoir une aide sociale. Malgré la situation économique plus mauvaise, la densité d'aide sociale en Allemagne de l'Est est (encore) inférieure à ce qu'elle est en Allemagne de l'Ouest (fig. 2). A celà on peut avancer plusieurs raisons. Tout d'abord, en raison du fort taux d'activité à l'époque de la RDA (notamment des femmes), la proportion de sansemplois ayant droit à l'allocation-chômage et de personnes âgées percevant une pension-retraite est plus importante qu'en Allemagne de l'Ouest. Deuxièmement, une proportion relativement forte de bénéficiaires de l'aide sociale est toujours intégrée dans le programme de l'office du travail et reçoit des indemnités salariales (le deuxième marché du travail). La régression de ce programme (tableau 1) se traduit (malgré la diminution, depuis 1997, du taux de chômage, même en Allemagne de l'Est) par une poursuite de la montée de la densité d'aide sociale en Allemagne de l'Est (fig. 1).

Tableau 1 : Statistiques du marché du travail en Allemagne de l'Ouest et en Allemagne de l'Est de 1991 à 1999 (a)

\begin{tabular}{|l|c|c|c|c|c|c|c|c|c|c|}
\hline & \multicolumn{5}{|c|}{$\begin{array}{c}\text { Allemagne de l'Ouest } \\
\text { (avec Berlin-ouest) }\end{array}$} & \multicolumn{5}{c|}{$\begin{array}{c}\text { Allemagne de l'Est } \\
\text { (sans Berlin-ouest) }\end{array}$} \\
\hline & 1991 & 1993 & 1995 & 1997 & 1999 & 1991 & 1993 & 1995 & 1997 & 1999 \\
\hline $\begin{array}{l}\text { Taux de chômage } \\
\text { des actifs du secteur civil }\end{array}$ & $6,3 \%$ & $8,2 \%$ & $9,3 \%$ & $11,0 \%$ & $9,9 \%$ & $10,3 \%$ & $15,8 \%$ & $14,9 \%$ & $19,5 \%$ & $19,0 \%$ \\
\hline $\begin{array}{l}\text { Nombre de chômeurs } \\
\text { en 1 000 }\end{array}$ & 1689 & 2270 & 2565 & 3021 & 2756 & 913 & 1149 & 1047 & 1364 & 1344 \\
\hline $\begin{array}{l}\text { Non compris } \\
\text { dans les chômeurs (en 1.000) }\end{array}$ & & & & & & & & & & \\
\hline $\begin{array}{l}\text { - Actifs bénéficiant } \\
\text { de mesures } \\
\text { de création d'emploi }\end{array}$ & 83 & 51 & 70 & 59 & 66 & 183 & 237 & 206 & 154 & 168 \\
\hline $\begin{array}{l}- \text { Personnes en formation } \\
\text { professionnelle }\end{array}$ & 349 & 349 & 303 & 245 & 215 & k.A. & 373 & 249 & 180 & 143 \\
\hline
\end{tabular}

(a) Données annuelles moyennes.

Source : Bundesanstalt für Arbeit.

16 Malgré de plus forts taux de chômage et un réajustement des mesures de l'office du travail, le fait que la densité d'aide sociale en Allemagne de l'Est soit toujours endessous de celle de l'Allemagne de l'Ouest pourrait en troisième lieu être le fait d'une plus forte "pauvreté cachée ", c'est à dire qu'il y a une plus grande proportion des ayants-droits à l'aide sociale qui ne sont pas au courant de leurs droits ou qui, pour 
différentes raisons, ne les font pas valoir (Deutscher Caritatsverband/ Diakonisches Werk 1997).

La comparaison de la densité d'aide sociale pour différentes classes d'âge en Allemagne de l'Est ou de l'Ouest montre de nettes différences de structure (fig. 4). Tandis que la densité d'aide sociale en Allemagne de l'Ouest diminue avec l'âge (voir chapitre II. B), en Allemagne de l'Est la densité d'aide sociale est particulièrement forte chez les moins de 7 ans et dans la classe des 18-29 ans. Ainsi, en Allemagne de l'Est, les jeunes adultes qui devraient en fait se trouver au commencement de leur vie professionnelle, sont manifestement les principaux perdants sur le marché du travail et sont particulièrement atteints par la régression du programme de l'office du travail. On peut vraisemblablement penser que les très fortes densités d'aide sociale chez les moins de 7 ans en Allemagne de l'Est sont en rapport avec le fait que les fortes valeurs des deux classes d'âge recouvrent la situation de familles entières. Le caractère explosif de cette situation vient du manque de perspectives pour beaucoup de jeunes gens en Allemagne de l'Est, et par là même des problèmes sociaux et psychiques croissant.

Figure. 4 : Comparaison des densités d'aide sociale (a) pour différentes classes d'âge en Allemagne de l'Ouest et de l'Est en 1997

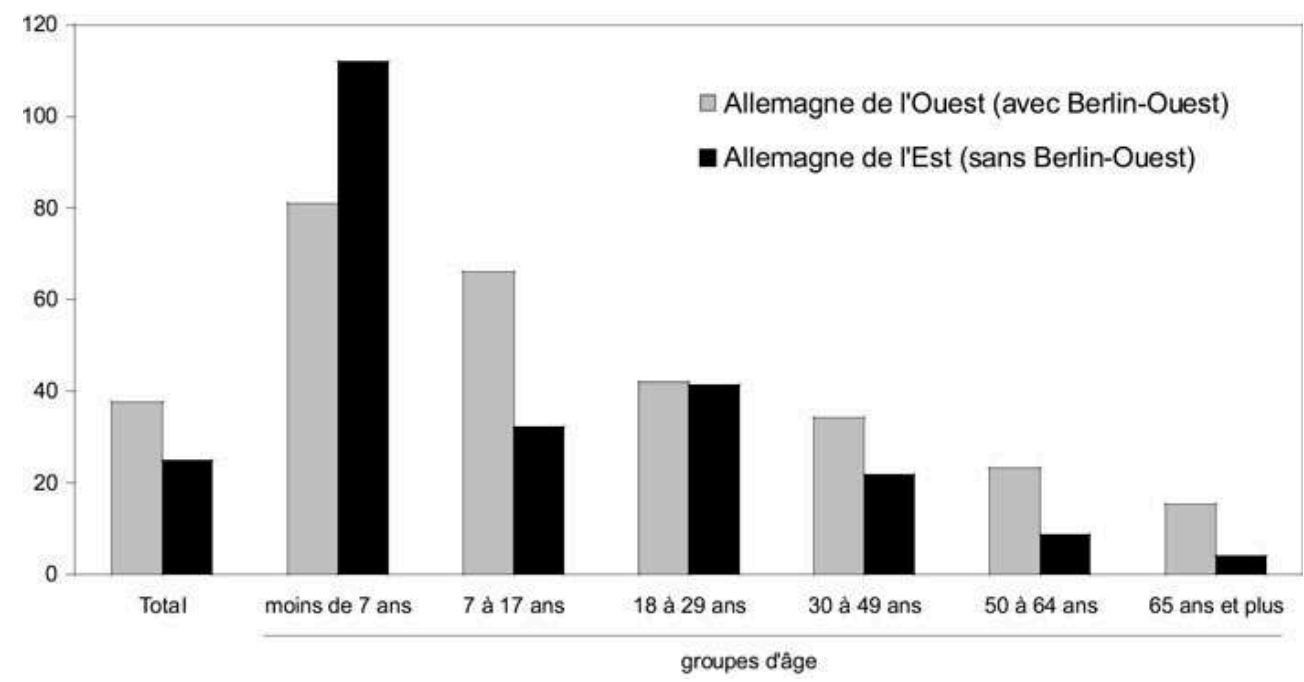

(a) Valeurs à la fin de l'année.

Source : Breuer et al. 1999, Statistisches Bundesamt, évaluations personnelles.

\section{B. Le gradient nord-sud}

Le contraste nord-sud constamment évoqué depuis le milieu des années 80 se rapportait d'abord et surtout aux disparités d'évolution économique en Allemagne de l'Ouest : ainsi, le taux de chômage en Allemagne du sud a tendance à être plus faible ; la proportion des branches en croissance et porteuses d'avenir par contre est plus élevée qu'en Allemagne du nord (Friedrichs et al. 1986). Mais ce gradient se reflète aussi dans la proportion des bénéficiaires de l'aide sociale qui est nettement plus faible en Allemagne du sud (notamment en Bavière et en Bade-Wurtemberg) que dans la plupart des régions du nord. En dehors des conditions économiques différentes, on explique souvent ce contraste par des différences d'évaluation et de comportement en fonction 
desquelles les bénéficiaires potentiels de l'aide sociale en Allemagne du sud font plus rarement valoir leurs droits.

L'étude empirique des disparités structurelles de la densité d'aide sociale dans les villes ouest-allemandes a largement confirmé ces réflexions (Klagge 1998, Klagge et Taubmann 1999). A partir d'études statistiques, on a pu isoler trois facteurs influençant à plus de $50 \%$ l'évolution au cours du temps des densités urbaines d'aide sociale et qui expliquent presque complètement le gradient nord-sud. Le taux de chômage est de loin le principal facteur. Le rapport entre la densité d'aide sociale et le degré de tertiarisation (avec un chômage constant) montre clairement que l'accentuation des différences sociales dans les villes à structure économique plus moderne va de pair avec des densités d'aide sociale plus fortes. Ce résultat permet de penser que les réflexions conduites sur l'évolution sociale dans ce que l'on appelle les "global cities» (Sassen 1991) sont en principe transposables aux villes plus petites, d'importance internationale moindre.

Un troisième facteur expliquant le contraste nord-sud joue un rôle certes moindre mais encore significatif. Il s'agit de l'influence plus forte dans le sud des milieux conservateurs. L'importance de ce facteur, qui a été établie à partir de la proportion des membres des églises (catholiques et évangéliques) ${ }^{4}$, ne doit cependant pas dissimuler les problèmes que pose son interprétation. Le fait que la densité d'aide sociale dans les milieux conservateurs soit plus basse que dans des milieux où le conservatisme est moins marqué peut résulter de plusieurs raisons. D'une part, il est plausible que les différences régionales d'évaluation, ainsi que les différences de comportement des ayants-droits à l'aide sociale qui en résultent, aient une influence sur la pauvreté cachée. Les raisons qui peuvent expliquer cette situation sont également variées et peuvent venir, non seulement de l'inégal degré de stigmatisation des bénéficiaires de l'aide sociale, mais aussi de l'importance des possibilités d'aides privées comme par exemple les réseaux familiaux et les églises. D'autre part, on ne peut pas non plus exclure que, dans les milieux conservateurs, la proportion de certaines classes à risques, comme par exemple les ménages monoparentaux, soit plus faible.

\section{Le contraste ville-campagne}

Un examen sélectif du gradient nord-sud en Allemagne de l'Ouest montre également de nettes différences internes à l'Allemagne du nord et à l'Allemagne du sud, pour lesquelles on peut parler de contraste ville-campagne, c'est à dire du gradient existant entre la ville et ses alentours. Une analyse des densités d'aide sociale a été faite sur la base des types de Kreis en fonction de leur degré d'urbanisation. Elle montre que les villes-centres, et en particulier celles se trouvant dans des agglomérations, présentent les plus hautes densités d'aide sociale (tableau 2). Celà est devenu également le cas en Allemagne de l'Est où, au début des années 90, les villes-centres (sans Berlin toutefois) présentaient même des densités d'aide sociale en moyenne plus basses que les Kreise ruraux (Klagge 1997) 
Tableau 2 : Densités d'aide sociale (a) en 1997 des villes en Allemagne de l'Ouest et en Allemagne de l'Est en fonction du degré d'urbanisation du Kreis

\begin{tabular}{|c|c|c|c|}
\hline & \multirow[b]{2}{*}{ Type de Kreis } & \multicolumn{2}{|c|}{ Densités d'aide sociale en 1997} \\
\hline & & $\begin{array}{l}\text { Allemagne de l'Ouest } \\
\text { (sans Berlin-ouest) }\end{array}$ & $\begin{array}{l}\text { Allemagne de l'Est } \\
\text { (avec Berlin-ouest) }\end{array}$ \\
\hline \multirow[t]{4}{*}{ Agglomérations } & Villes-centres & 57 & 63 \\
\hline & Kreis à très forte densité & 27 & 16 \\
\hline & Kreis à forte densité & 27 & 18 \\
\hline & Kreis rural & 35 & 19 \\
\hline \multirow[t]{3}{*}{$\begin{array}{l}\text { Espaces en voie } \\
\text { d'urbanisation }\end{array}$} & Villes-centres & 56 & 33 \\
\hline & Kreis à forte densité & 29 & 21 \\
\hline & Kreis rural & 26 & 20 \\
\hline \multirow[t]{2}{*}{ Espaces ruraux } & Kreis à forte densité & 25 & 25 \\
\hline & Kreis à faible densité & 22 & 23 \\
\hline
\end{tabular}

(a) Valeurs à la fin de l'année.

Source : BBR 1999

Les fortes proportions d'aide sociale dans les villes-centres s'expliquent par les forts taux de chômage, et surtout par les concentrations de classes à fort risque de paupérisation comme les ménages monoparentaux, les familles nombreuses et les étrangers. L'alignement de la situation en Allemagne de l'Est sur celle de l'Allemagne de l'Ouest se comprend par l'important processus de suburbanisation, en particulier dans les régions de Berlin et de Leipzig. Tandis qu'au début des années 90 les différences de structure socio-démographique des ménages dans les régions urbaines et rurales étaient plutôt faibles, les processus de suburbanisation liés à l'accentuation des différences sociales ont entrainé une différenciation spatiale. Les très basses densités d'aide sociale dans les Kreise entourant les villes-centres, c'est à dire dans les Kreise à (très) forte densité, dans les Kreise ruraux des régions urbanisées et, dans une moindre mesure, dans les régions en voie d'urbanisation, confirment cette explication.

\section{Conclusion}

L'analyse des disparités spatiales de la pauvreté et de l'importance de l'aide sociale a montré qu'en dehors des faits économiques et de la présence de classes à risques, on doit considérer que les différences de comportement ainsi que l'influence des réseaux sociaux sont des facteurs exerçant une influence importante. On dispose d'arguments montrant qu'il y a des façons différentes de surmonter la pauvreté, aussi bien à l'intérieur d'une même ville que d'une région à l'autre, et peut-être même dans les différentes catégories de population.

D'un point de vue méthodologique, cela est d'autant plus significatif que la pauvreté prise en compte par l'aide sociale ne recouvre pas complètement l'insuffisance de revenus, mais, en raison de l'absence de prise en considération de la "pauvreté cachée », qu'elle ne représente en fait qu'une approximation. Par ailleurs, dans la perspective de nouvelles recherches, on doit remarquer que tous les résultats obtenus ici sont basés sur des données récoltées par unité spatiale et reposent sur les rapports relatifs à la société et à la pauvreté ainsi que sur les déclarations des experts. Afin de parvenir à des données plus sûres, il serait nécessaire de ne pas s'en tenir seulement aux estimations qualitatives des thèmes considérés, mais aussi d'analyser des données isolées, si possible en coupes transversales et longitudinales. Pour une interprétation 
plus poussée du contenu de ces études, ainsi que du point de vue de la politique sociale, il serait important de rechercher les conditions et les motivations qui conduisent éventuellement à la renonciation à l'aide sociale, ainsi que de mettre en évidence les conséquences financières et sociales pour les personnes concernées. Dans ce contexte, il est particulièrement intéressant de se poser la question du rôle que les réseaux sociaux peuvent jouer pour surmonter et/ou même éviter la pauvreté, et comment par exemple le travail social de quartier, plus actif au cours de la période récente, peut utiliser ces potentialités.

De toute façon, la portée des mesures visant à combattre la pauvreté ne doit pas être surestimée. En outre, les inflexions fondamentales dans les domaines de la politique du logement et de l'emploi, ainsi que la réforme du système de l'assurance sociale sont d'une plus grande importance pour l'ampleur future, la répartition spatiale et la concentration de la pauvreté ainsi que pour leurs conséquences sociales. Si l'amélioration annoncée sur le marché du travail devait effectivement représenter un tournant et entraîner un recul à long terme du nombre des chômeurs, alors, pour la première fois depuis 30 ans, il pourrait y avoir de nouveau une diminution durable des taux de pauvreté.

\section{BIBLIOGRAPHIE}

BBR (Bundesamt für Bauwesen und Raumordnung) (1999). - Aktuelle Daten zur Entwicklung der Städte, Kreise und Gemeinden. Ausgabe 1999, BBR-Berichte 3, BBR, Bonn.

Bieback K.-J., Milz H. (Hrsg.) (1995). - Neue Armut. Campus, Frankfurt / New York.

Breuer W., Engels D, ISG Sozialforschung und Gesellschaftspolitik GmbH (1999). Grundinformationen und Daten zur Sozialhilfe, Informationsschrift im Auftrag des Bundesministeriums für Arbeit und Sozialordnung, Bonn (abzurufen unter : www. bma. bund. de, im August 2000).

Büchel F., Frick J., Voges, W. (1997) - Der Sozialhilfebezug von Zuwanderern in Westdeutschland. In : Kölner Zeitschrift fuer Soziologie und Sozialpsychologie 49, pp. 272-290.

Deutscher Caritasverband, Diakonisches Werk (1997). - Menschen im Schatten. Erfahrungen von Caritas und Diakonie in den neuen Bundesländern. Untersuchung der Lebenslagen der Rat- und Hilfesuchenden in den offenen Diensten von Caritas und Diakonie in den neuen Bundesländern, Karlsruhe/Stuttgart.

Friedrichs J., Häussermann H., Siebel W. (Hrsg.) (1986). - Süd-Nord-Gefälle in der Bundesrepublik? Sozialwissenschaftliche Analysen, Westdeutscher Verlag, Opladen.

Geissler R. (1996). - Die Sozialstruktur Deutschlands. Zur gesellschaftlichen Entwicklung mit einer Zwischenbilanz zur Vereinigung, Westdeutscher Verlag, Opladen 1996, 2, neubearbeitete und erweiterte Auflage.

Hanesch W. et al. (1994). - Armut in Deutschland. Der Armutsbericht des DGB und des Paritätischen Wohlfahrtsverbands, Rowohlt, Reinbek b. Hamburg. 
Hartmann H. (1981). - Sozialhilfebedürftigkeit und « Dunkelziffer der Armut » . Bericht über das Forschungsprojekt zur Lage potentiell Sozialhilfeberechtigter, Schriftenreihe des Bundesministers für Jugend, Familie und Gesundheit Band 98, Kohlhammer, Stuttgart/Berlin / Köln /Mainz.

Klagge B. (1997). - Armut in Deutschland. Disparitäten zwischen Ost und West, Nord und Süd, Stadt und Land. In : Geographie Heute 18, Heft 156, pp. 10-15.

Klagge B. (1998). - Armut in westdeutschen Städten. Ursachen und Hintergründe der Disparitäten städtischer Armutsraten. In : Geographische Rundschau 50, pp. 139-145.

Klagge B., Taubmann W. (1999). - Armut in den Städten der Bundesrepublik Deutschland. Ausmaß, Strukturen fund räumliche Ausprägungen. Endbericht des DFG-Forschungsprojektes Ta 49/11-1 an der Universität Bremen.

Kronauer M. (1997) - « Soziale Ausgrenzung » und « Underclass ». Über neue Formen der gesellschaftlichen Spaltung. In : Leviathan 25, pp. 28-49.

Leibfried S. et al. (1995) - Zeit der Armut, Suhrkamp, Frankfurt.

Neuhäuser J. (1996) - Sozialhilfe und Leistungen an Asylbewerber 1994. Ausgaben und Einnahmen. In : Wirtschaft und Statistik, H. 10/1996, pp. 633-641.

Sassen S. (1991) - The global city : New York, London, Tokyo, Princeton University Press, Princeton, NJ.

Wendt, H. (1995) - Asylbewerber in Deutschland. In : Geographische Rundschau 47, pp. 443-446.

\section{NOTES}

1. Une étude empirique actuelle des disparités intra-urbaines dans les villes ouest-allemandes se trouve dans Klagge et Taubmann (1999); pour une discussion théorique contenant des comparaisons internationales des processus de ségrégation et d'exclusion, voir Kronauer (1997).

2. Les données annuelles cumulées concernent tous les bénéficiaires de l'aide sociale au cours d'une année, tandis que les données journalières sont relatives à une période précise, en règle générale la fin de l'année (31.12). En conséquence, les données annuelles cumulées sont nettement au-dessus des valeurs correspondantes de fin d'année.

3. Cela pose problème parce qu'au cours de la période, la forte variation du nombre des demandeurs d'asile n'a pas été sans influencer notablement les densités d'étrangers bénéficiant d'une aide sociale (Klagge 1998).

4. Les membres des églises acquittant l'impôt sur la base du concordat signé entre l'Allemagne et le Saint-Siège.

5. La très forte valeur pour les villes-centres des agglomérations d'Allemagne de l'Est en 1997 est déterminée par l'adjonction de Berlin (avec une densité d'aide sociale de 78) ; les autres villescentres est-allemandes situées dans des agglomérations présentent, à l'exception de Dresde, des valeurs nettement plus faibles, quoique relativement élevées (Leipzig: 32, Chemnitz: 26, Potsdam : 24, Dresde : 15). 


\section{RÉSUMÉS}

Les changements subis par les structures économiques et sociales, au cours des trente dernières années, ont influencé d'une manière décisive l'évolution et la structure de la population pauvre en Allemagne. La montée du chômage depuis 1970 s'accompagne d'une croissance de la pauvreté qui, dans le cadre de la présente étude, est calculée à partir de l'importance des versements de l'aide sociale. En-dehors des chômeurs, ce sont surtout les étrangers et les enfants qui sont devenus les catégories les plus menacées par la pauvreté qui s'est développée au cours de cette phase de profonde mutation démographique. Le but essentiel de cette contribution est la représentation et l'analyse empirique des disparités spatiales de l'aide sociale; les thèmes retenus sont les gradients est-ouest et nord-sud, ainsi que les contrastes ville-campagne. Les facteurs déterminants des disparités existantes sont non seulement la proportion que représentent les groupes à risque dans la population, mais aussi les mesures politiques (en particulier la politique de l'emploi en Allemagne de l'Est) et les différences d'appréciation et de comportement face à la pauvreté. Dans l'ensemble, le modèle de répartition des disparités reste stable. L'évolution la plus notable est enregistrée en Allemagne de l'Est où les disparités de pauvreté entre ville et campagne se sont fortement rapprochées de celles existant en Allemagne de l'Ouest dans les années 90.

Economic and social structural change in the last 30 years have had a decisive impact on the development and structure of the poor in Germany. Rising unemployment since 1970 has been accompanied by growing poverty rates, which in this contribution are based on welfare recipiency rates. Apart from the unemployed, foreigners and children have become special groups with a high risk of poverty, partly as a consequence of deep structural changes in German demography. The main focus of this contribution is the description and analysis of spatial disparities of poverty in Germany, especially the east-west and the north-south divides as well as urban-ruralcontrasts. The important determinants of these differences are not only proportion of risk groups in the respective populations, but also policies (esp. labor market policies in East Germany) and behavioral differences. The observed patterns of disparity are quite stable over time. The most dynamic development is taking place within East Germany, where in the 1990s the differences in powerty between town and country has become more and more like that of West Germany in the 1990s.

Wirtschaftlicher und sozialer Strukturwandel in den letzten 30 Jahren haben die Entwicklung und Struktur der Armutsbevölkerung in Deutschland entscheidend beeinflußt. Der Anstieg der Arbeitslosigkeit seit 1970 geht mit einer wachsenden Armut einher, die im vorliegenden Beitrag über den Bezug von Sozialhilfe operationalisiert wird. Neben Arbeitslosen haben sich im Zuge tiefgreifender demographischer Veränderungsprozesse insbesondere Ausländer und Kinder zu besonderen Risikogruppen der Armut entwickelt. Den Schwerpunkt des Beitrags bildet die Darstellung und empirische Analyse regionaler Disparitäten des Sozialhilfebezugs; thematisiert wird neben dem West-Ost- und dem Nord-Süd-Gefälle der Stadt-Land-Gegensatz. Wichtige Determinanten der bestehenden Disparitäten sind neben dem Anteil von Risikogruppen an der Bevölkerung auch politische Maßnahmen (insbesondere Arbeitsmarktpolitik in Ostdeutschland) und Unterschiede in Wertvorstellungen und Verhaltensweisen. Insgesamt ist das Muster der Disparitäten relativ stabil. Die größte Entwicklungsdynamik ist innerhalb Ostdeutschlands festzustellen, wo sich die Armutsdisparitäten zwischen Stadt und Land denen in Westdeutschland in den 1990er Jahren stark angeglichen haben. 
INDEX

Mots-clés : Allemagne, disparités régionales, pauvreté, versement de l'aide sociale

Schlüsselwörter : Armut, Deutschland, regionale Disparitäten, Sozialhilfe (bezug)

Keywords : Germany, poverty, regional disparities, welfare recipiency (rates)

\section{AUTEURS}

BRITTA KLAGGE

Institut für Geographie - der Universität Hamburg - Bundesstrasse 55, 20146 Hamburg - Tel.

0049-40-42838-4968, Fax -4981 - klagge@geowiss.uni-hamburg.de 